\title{
Prevalence of ketosis, ketonuria, and ketoacidosis during liberal glycemic control in critically ill patients with diabetes: an observational study
}

Nora Luethi ${ }^{1}$, Luca Cioccari ${ }^{1,2}$, Marco Crisman ${ }^{1,3}$, Rinaldo Bellomo ${ }^{1,4}$, Glenn M. Eastwood ${ }^{1}$ and Johan Mårtensson ${ }^{1,5^{*}}$

\begin{abstract}
Background: It is uncertain whether liberal glucose control in critically ill diabetic patients leads to increased ketone production and ketoacidosis. Therefore, we aimed to assess the prevalence of ketosis, ketonuria and ketoacidosis in critically ill diabetic patients treated in accordance with a liberal glycemic control protocol.
\end{abstract}

Methods: We performed a prospective observational cohort study of 60 critically ill diabetic patients with blood and/or urine ketone bodies tested in ICU. All patients were treated according to a liberal glucose protocol targeting a blood glucose level (BGL) between 10 and $14 \mathrm{mmol} / \mathrm{l}$ in a single tertiary intensive care unit in Australia. We measured quantitative bedside blood 3-beta-hydroxybutyrate ( $\beta$-OHB) and semi-quantitative urine ketones on ICU admission and daily during ICU stay, for a maximum of 10 consecutive days.

Results: Median blood $\beta$-OHB level on admission was $0.3(0.1,0.8) \mathrm{mmol} / \mathrm{l}$. Ketoacidosis was rare $(3 \%)$, but some level of ketosis ( $\beta-O H B \geq 0.6 \mathrm{mmol} / \mathrm{l}$ ) was found in 38 patients ( $63 \%)$ early during their ICU stay. However, there was no significant difference in prevalence or severity of ketonemia and ketonuria among patients with $B G L$ above (permissive hyperglycemia) or below $10 \mathrm{mmol} / \mathrm{l}$. On multivariable linear regression analysis there was no association between blood ketone levels and BGL, HbA1c, lactate levels, hematocrit, catecholamine infusion or APACHE III score. In contrast, blood ketone levels tended to be higher after cardiopulmonary bypass surgery $(P=0.06)$.

Conclusions: Liberal glycemic control in critically ill diabetic patients does not appear to be associated with a high prevalence of ketoacidosis or ketonemia. Moreover, ketosis is typically present on admission and resolves rapidly. Finally, cardiopulmonary bypass surgery may be an important trigger of ketone body production.

Trial registration: Australian New Zealand Clinical Trials Registry (ACTRN12615000216516; trial registration date 5 March 2015).

Keywords: Ketone bodies, 3-beta-hydroxybutyrate, Diabetes mellitus, Critical illness, Permissive hyperglycemia, Adults

\footnotetext{
* Correspondence: johan.martensson@austin.org.au

${ }^{1}$ Department of Intensive Care, Austin Hospital, 145 Studley Rd, Heidelberg 3084, VIC, Australia

${ }^{5}$ Department of Anaesthesia and Intensive Care Medicine, Karolinska

University Hospital, Department of Physiology and Pharmacology, Karolinska

Institutet, 17177 Stockholm, Sweden

Full list of author information is available at the end of the article
} 


\section{Background}

Current guidelines recommend using insulin to maintain a blood glucose level (BGL) between 6 and $10 \mathrm{mmol} / \mathrm{l}$ in critically ill patients [1-4]. However, controlling glucose within this target range is associated with a relatively high rate of hypoglycemia [5] and increased mortality [6] in diabetic patients especially if adapted to chronic hyperglycemia (glycated hemoglobin A1c [HbA1c] $>7 \%$ ). Therefore, a more liberal glycemic approach (target BGL between 10 and $14 \mathrm{mmol} / \mathrm{l}$ ) may be safer in patients with diabetes. Recent pilot studies have demonstrated that such "permissive hyperglycemia" effectively reduces the need for exogenous insulin administration [7]. Consequently, in response to the introduction of a liberal glucose protocol in diabetic patients, the incidence of both absolute hypoglycemia (BGL $<3.9 \mathrm{mmol} / \mathrm{l}$ ) and potentially harmful relative hypoglycemia (an acute and marked decrease in glycaemia) may be reduced.

However, whether such liberal strategies lead to relative insulin deficiency, accelerated ketone body production and induce ketoacidosis is uncertain. In addition to insulin deficiency, several other factors may stimulate ketogenesis via beta-oxidation of free fatty acids (FFA) in critically ill patients. Such factors include fasting (a ubiquitous state in patients admitted to the intensive care unit [ICU]), increased secretion of counter-regulatory hormones (glucagon, adrenaline, growth hormone and cortisol), administration of exogenous catecholamines (adrenaline and noradrenaline) [8] and administration of parenteral nutrition containing medium-chain triglycerides [9]. Furthermore, entry of acetyl coenzyme A into the Krebs cycle may be impaired during critical illness due to reduced oxaloacetate levels. Consequently, more acetyl coenzyme will be used for synthesis of ketone bodies [10]. Similarly, elevated levels of deaminated amino acids may feed ketogenesis. Moreover, although the mechanism is less clear, alkalosis appears to stimulate ketogenesis, particularly in diabetic subjects [11]. Finally, elevated blood ketone levels have been observed following cardiopulmonary bypass surgery, especially when performed under hypothermia [12]. Although, many of these factors may co-exist in the ICU setting, their combined impact on ketone body production is unknown.

Accordingly, we conducted a prospective observational study to explore the incidence of ketosis, ketonuria and ketoacidosis during liberal glucose management of critically ill diabetic patients. In addition, we aimed to assess the association of chronic glycaemia (HbA1c), acute glycaemia, illness severity, alkalosis, catecholamine infusion and cardiopulmonary bypass surgery with blood ketone levels on ICU admission in such patients.

\section{Methods}

\section{Data collection}

We performed a prospective observational study in all diabetic patients admitted to a single tertiary adult ICU between 30 August and 17 November 2015. All patients were treated in accordance with our local protocol for diabetic patients targeting a blood glucose levels (BGL) between 10 and $14 \mathrm{mmol} / \mathrm{l}$ and in which BGLs above $14 \mathrm{mmol} / \mathrm{l}$ were treated with intravenous or subcutaneous insulin. The Austin Health Human Research Ethics Committee approved the study (approval number LNR/14/Austin/487, HREC 131217 and 120712) with a waiver for informed consent. The study was performed in accordance with the ethical standards laid down in the 1964 Declaration of Helsinki and its later amendments.

For the purpose of this study, we excluded patients admitted with diabetic ketoacidosis (DKA) or a hyperglycemic hyperosmolar state (HHS). We collected demographic data, ICU admission diagnoses, type of diabetes, insulin dependency, chronic medication and Acute Physiology and Chronic Health Evaluation (APACHE) III score. We obtained daily data on use of renal replacement therapy (RRT), incidence of acute liver failure, rate of adrenaline and noradrenaline infusion, type and rate of enteral or parenteral nutrition and insulin administration.

\section{Blood and urine sample collection and analysis}

We collected arterial blood gas (ABG) data and spot urine samples for measurement of blood and urine ketone levels as soon as possible after ICU admission. Thereafter, we performed daily ketone measurement early in the morning, which likely represents peak ketone levels [13] and simultaneously tested urine samples for ketonuria until ICU discharge or up to a maximum of 10 days. We measured blood $\beta$ hydroxybutyrate $(\beta-\mathrm{OHB})$ using the Freestyle Optium Xceed point-of-care meter (Abbott Diabetes Care Inc., Maidenhead, UK). Simultaneously, we measured hematocrit since previous data suggest that point-ofcare platforms may overestimate $\beta-\mathrm{OHB}$ at subnormal hematocrit levels [14]. Ketonuria was semi-quantified using Combur-test ${ }^{\circ}$ strips (Roche Diagnostics, Rotkreuz, Switzerland). ABG samples were analyzed using the Radiometer ABL825 blood gas analyzer (Radiometer Medical A/S, Brønshøj, Denmark). We analysed HbA1c on ICU admission at our central laboratory, using COBAS INTEGRA ${ }^{\circ} 800$ (Roche Diagnostics, Indianapolis, IN, USA).

\section{Definitions}

We categorized ketosis according to blood $\beta$-OHB levels as absent $(<0.6 \mathrm{mmol} / \mathrm{l})$, mild $(0.6-1.5 \mathrm{mmol} / \mathrm{l})$, moderate 
$(1.6-3 \mathrm{mmol} / \mathrm{l})$ or marked $(>3 \mathrm{mmol} / \mathrm{l})$. Ketonuria was semi-quantified as mild, moderate or marked for urine ketone levels of $1-4 \mathrm{mmol} / \mathrm{l}, 5-14 \mathrm{mmol} / \mathrm{l}$ and $\geq 15 \mathrm{mmol} / \mathrm{l}$, respectively. The presence of ketoacidosis was assessed using both definitions suggested by the American Diabetes Association (ADA) and the Joint British Diabetes Society (JBDS) $[15,16]$.

\section{Statistical analysis}

We analyzed data using STATA $^{\circ}$ version Stata/SE 13.1 (Stata Corp., College Station, TX, USA). Continuous variables were expressed as median (interquartile range [IQR]) and categorical variables as $n$ (\%). We compared continuous data using the MannWhitney $U$ test (two groups) or the Kruskal-Wallis test (multiple groups). For categorical variables, we used the chi-squared test or the Fisher's exact test. The Spearman's rank correlation coefficient was calculated to assess correlations. Changes over time for BGLs and blood ketone levels during permissive hyperglycemia (BGL $10-14 \mathrm{mmol} / \mathrm{l}$ ) were tested by repeated measures analysis of variance (RM-ANOVA) using ICU day as the repeated measures variable. For comparison of these variables' change over time in patients who did and did not receive insulin, an interaction variable (between category [insulin therapy, yes vs. no] and time) was introduced in the RM-ANOVA model. We used multivariable linear regression analysis to assess the relationship between blood ketone levels on ICU admission and the following variables: APACHE III score (tertiles), ongoing catecholamine infusion (yes vs. no), HbAlc, admission BGL, cardiopulmonary bypass surgery (yes vs. no), admission hematocrit and admission blood lactate level. Since insulin therapy may impact both ketogenesis and BGLs we restricted the multivariable linear regression analysis to patients not receiving insulin. A two-sided $P$ value $<0.05$ was considered statistically significant.

\section{Results}

\section{Baseline characteristics}

We studied 60 consecutive critically ill diabetic patients (17 [28\%] females) with a median (IQR) age of $66(60,74)$ years and a median APACHE III score of 60 (46, 76). Overall, 57 (95\%) patients had type 2 diabetes of whom 24 (42\%) were insulin-dependent prior to ICU admission. Two patients were treated with a sodium-glucose cotransporter-2 inhibitor before ICU admission; none developed ketosis or ketonuria in ICU. The majority (42\%) of patients were admitted after cardiovascular surgery, of which 22 patients underwent cardiopulmonary bypass surgery (during normothermia in all 22 patients). Three (5\%) patients were admitted with acute liver failure and one with acute alcohol intoxication. Four patients had a history of chronic alcohol abuse.

A total of 46 (77 \%) patients had a peak BGL $\geq 10 \mathrm{mmol} / \mathrm{l}$ during ICU admission. Compared to patients with a peak BGL $<10 \mathrm{mmol} / \mathrm{l}(\mathrm{n}=14)$, patients with a peak BGL $\geq 10 \mathrm{mmol} / \mathrm{l}$ were younger $(P=$ $0.02)$, had higher HbA1c $(7.0[6.2,7.8] \%$ vs. 6.2 [5.9, $6.5] \%, P=0.009)$ and greater admission BGL $(P=$ $0.01)$. Moreover, a higher proportion of patients with peak BGL $\geq 10 \mathrm{mmol} / \mathrm{l}$ were prescribed chronic insulin therapy (23 [50\%] patients vs. $1[7 \%]$ patient, $P=0.007$ ). However, we observed no significant differences in APACHE III score, admission blood ketone levels, admission blood lactate levels or hematocrit levels between the two groups. A total of 21 (46\%) patients with peak BGL $\geq 10 \mathrm{mmol} / \mathrm{l}$ and two (14 \%) patients with peak BGL $<10 \mathrm{mmol} / \mathrm{l}$ received catecholamine infusion on admission $(P=0.06)$. Admission alkalosis $(\mathrm{pH}>7.45)$ was relatively uncommon and only six (10\%) patients received enteral or parenteral nutrition on admission. Median length of stay in ICU (3.7 [2.2, 6.2] days) and in hospital (11 [6.7, 24] days) as well as ICU (8 \%) and hospital $(10 \%)$ mortality was similar in the two groups (Table 1).

\section{Glycemic control, ketonemia and ketonuria}

We analysed 280 blood glucose measurements, 277 blood ketone measurements, 261 urine ketone measurements and 276 hematocrit measurements obtained within $8.3(1.6,12.0)$ hours after ICU admission and daily until ICU discharge or day 10, whichever came first. A total of 274 (99\%) hematocrit levels were below normal $(<40 \%)$. Furthermore, hematocrit decreased significantly over a maximum observation period of 10 days in ICU (Additional file 1: Figure S1).

Overall, 27 of 46 (59 \%) patients with a peak BGL $\geq 10 \mathrm{mmol} / \mathrm{l}$ required insulin to maintain their BGLs between 10 and $14 \mathrm{mmol} / \mathrm{l}$. Of these 27 patients, $74 \%$ had preexisting insulin-dependent diabetes. In addition, 27 of 46 (59 \%) patients with a peak BGL $\geq 10 \mathrm{mmol} / \mathrm{l}$ received enteral and/or parenteral nutrition during ICU admission whereas only two (14\%) patients with a peak BGL $<10 \mathrm{mmol} / \mathrm{l}$ received such nutrition. Mean total caloric intake progressively increased during ICU admission (Additional file 1: Figure S2). Only one patient received parenteral nutrition, which did not contain mediumchain triglycerides (OliClinomel, Baxter Healthcare, Deerfield, IL, USA). Insulin was not administered to patients with a peak BGL $<10 \mathrm{mmol} / \mathrm{l}$ in ICU. Half of all patients received catecholamine infusion (mainly noradrenaline) while in ICU (Table 2).

Overall, 38 (63\%) patients showed some degree of ketosis $(\beta-\mathrm{OHB} \geq 0.6 \mathrm{mmol} / \mathrm{l})$ during ICU admission (20 
Table 1 Baseline characteristics and outcomes for all diabetic patients and according to peak blood glucose level

\begin{tabular}{|c|c|c|c|c|}
\hline Characteristic & All patients & Peak BGL $\geq 10 \mathrm{mmol} / \mathrm{l}$ & Peak BGL <10 mmol// & $P$ value \\
\hline Number of patients & $60(100)$ & $46(77)$ & $14(23)$ & \\
\hline Age, years & $66(60,74)$ & $65(56,71)$ & $74(65,80)$ & 0.02 \\
\hline Female sex, n (\%) & $17(28)$ & $15(33)$ & $2(14)$ & 0.31 \\
\hline Weight, kg ( $n=58)$ & $84(74,100)$ & $84(78,104)$ & $83(74,100)$ & 0.39 \\
\hline $\mathrm{BMI}, \mathrm{kg} / \mathrm{m}^{2}(\mathrm{n}=40)$ & $31(27,34)$ & $32(28,35)$ & $28(24,31)$ & 0.11 \\
\hline APACHE III score & $60(46,76)$ & $59(46,75)$ & $62(48,79)$ & 0.71 \\
\hline Type 1 diabetes, n (\%) & $3(5)$ & $3(7)$ & 0 & \\
\hline Type 2 diabetes, n (\%) & $57(95)$ & $43(93)$ & $14(100)$ & 1.00 \\
\hline Type 2 diabetes treatment, n (\%) & & & & 0.007 \\
\hline Diet only & $8(13)$ & $5(11)$ & $6(43)$ & \\
\hline Oral agent(s) only & $25(42)$ & $18(39)$ & $7(50)$ & \\
\hline Insulin only & $11(18)$ & $10(22)$ & $1(7)$ & \\
\hline Insulin + oral agent(s) & $13(22)$ & $13(28)$ & 0 & \\
\hline Non-operative admission diagnosis, n (\%) & & & & 0.59 \\
\hline Cardiovascular & $6(10)$ & $5(26)$ & $1(14)$ & \\
\hline Sepsis & $6(10)$ & $5(26)$ & $1(14)$ & \\
\hline Respiratory & $6(10)$ & $3(16)$ & $3(43)$ & \\
\hline Renal/metabolic & $6(10)$ & $5(26)$ & $1(14)$ & \\
\hline Other & $2(3)$ & $1(5)$ & $1(14)$ & \\
\hline Operative admission diagnosis, n (\%) & & & & 0.09 \\
\hline Cardiovascular & $25(42)$ & $20(74)$ & $5(71)$ & \\
\hline Gastrointestinal & $6(10)$ & $6(22)$ & $1(14)$ & \\
\hline Other & $3(5)$ & $1(4)$ & $1(14)$ & \\
\hline $\mathrm{HbA} 1 \mathrm{c}$ on ICU admission, \% & $6.7(6.0,7.7)$ & $7.0(6.2,7.8)$ & $6.2(5.9,6.5)$ & 0.009 \\
\hline Blood glucose on ICU admission, mmol/I & $9.3(7.6,12)$ & $10(7.8,13)$ & $8.1(7.4,9.0)$ & 0.01 \\
\hline Blood ketones on ICU admission, mmol/I & $0.3(0.1,0.8)$ & $0.3(0.1,0.8)$ & $0.4(0.2,0.6)$ & 0.99 \\
\hline Ketosis on ICU admission, n (\%) & $23(39)$ & $17(38)$ & $6(43)$ & 0.12 \\
\hline Ketonuria on ICU admission, $n(\%)(n=57)$ & $8(14)$ & $6(13)$ & $2(17)$ & 0.09 \\
\hline Lactate on ICU admission, mmol// & $1.6(1.1,2.3)$ & $1.7(1.2,2.7)$ & $1.2,(1.0,2.0)$ & 0.06 \\
\hline Hematocrit on ICU admission, \% & $27(25,31)$ & $27(25,29)$ & $32(27,35)$ & 0.07 \\
\hline Alkalosis on ICU admission ${ }^{\mathrm{a}}$, n (\%) & $7(12)$ & $7(15)$ & 0 & \\
\hline Catecholamine infusion on ICU admission, n (\%) & $23(38)$ & $21(46)$ & $2(14)$ & 0.06 \\
\hline Nutrition on ICU admission, n (\%) & $6(10)$ & $6(13)$ & 0 & \\
\hline Acute liver failure, n (\%) & $3(5)$ & $2(4)$ & $1(7)$ & 0.56 \\
\hline Chronic alcohol abuse, n (\%) & $4(7)$ & $3(7)$ & $1(7)$ & 1.00 \\
\hline Acute alcohol intoxication, n (\%) & $1(2)$ & $1(2)$ & 0 & \\
\hline ICU length of stay, days & $3.7(2.2,6.2)$ & $3.7(2.3,6.9)$ & $3.4(2.1,3.8)$ & 0.26 \\
\hline Hospital length of stay, days & $11(6.7,24)$ & $11(7.2,23)$ & $13(5.4,24)$ & 0.86 \\
\hline ICU mortality, n (\%) & $5(8)$ & $4(9)$ & $1(7)$ & 0.85 \\
\hline Hospital mortality, n (\%) & $6(10)$ & $5(11)$ & $1(7)$ & 0.68 \\
\hline
\end{tabular}

Values are median (IQR) or $\mathrm{n}(\%)$

$B G L$ blood glucose level, BMI body mass index, APACHE Acute Physiology and Chronic Health Evaluation, ICU intensive care unit ${ }^{\mathrm{a}} \mathrm{pH}>7.45$ 
Table 2 Glycemic control, ketonemia, ketonuria and acid-base status of diabetic patients with and without permissive hyperglycemia

\begin{tabular}{|c|c|c|c|c|}
\hline Variable & All patients & $\begin{array}{l}\text { Peak BGL } \\
\geq 10 \mathrm{mmol} / \mathrm{l}\end{array}$ & $\begin{array}{l}\text { Peak BGL } \\
<10 \mathrm{mmol} / \mathrm{l}\end{array}$ & $P$ value \\
\hline Number of patients, $\mathrm{n}(\%)$ & $60(100)$ & $46(77)$ & $14(23)$ & \\
\hline No. of blood glucose measurements, n (\%) & 280 & 229 & 51 & \\
\hline No. of blood glucose measurements per patient & $4(3,7)$ & $4(3,7)$ & $3(2,5)$ & 0.19 \\
\hline Peak glucose level, mmol/l & $12.0(10.0,15.0)$ & $14(12,17)$ & $8.2(7.4,9.5)$ & $<0.001$ \\
\hline Mean glucose level, mmol/l & $10.0(8.3,13.0)$ & $12(9.5,13)$ & $7.1(6.6,8.3)$ & $<0.001$ \\
\hline Min glucose level, mmol/l & $8.0(6.2,9.4)$ & $8.8(7,9.9)$ & $6.2(5.7,7.5)$ & 0.007 \\
\hline No. of blood ketone measurements, n (\%) & 277 & 228 & 49 & \\
\hline No. of blood ketone measurements per patient & $4(3,7)$ & $4(3,7)$ & $3(2,4)$ & 0.11 \\
\hline Time between ICU admission until first measurement, hours & $8.3(1.6,12)$ & $8.9(1.7,13)$ & $3.2(1.5,11)$ & 0.02 \\
\hline Patients receiving insulin, $\mathrm{n}(\%)$ & $27(45)$ & $27(59)$ & 0 & \\
\hline Intravenous insulin rate, units/h & $2(1,2)$ & $2(1,2)$ & 0 & \\
\hline Patients receiving nutrition, $\mathrm{n}(\%)$ & $29(48)$ & $27(59)$ & $2(14)$ & \\
\hline Enteral nutrition, n (\%) & $28(47)$ & $26(57)$ & $2(14)$ & \\
\hline Total parenteral nutrition, $\mathrm{n}(\%)$ & $2(3.3)$ & $2(4.3)$ & 0 & \\
\hline Patients receiving catecholamine infusion, n (\%) & $30(50)$ & $27(46)$ & $3(21)$ & 0.03 \\
\hline Noradrenaline, n (\%) & $29(48)$ & $26(57)$ & $3(21)$ & 0.03 \\
\hline Maximum noradrenaline rate, $\mathrm{\mu g} / \mathrm{min}$ & $5.0(2.0,16)$ & $7.5(2.0,16)$ & $2.0(1.0,4.0)$ & 0.11 \\
\hline Adrenaline, n (\%) & $7(12)$ & $7(15)$ & 0 & \\
\hline Maximum adrenaline rate, $\mu \mathrm{g} / \mathrm{min}$ & $3.0(2.0,4.0)$ & $3.0(2.0,4.0)$ & NA & \\
\hline Ketosis episodes >0.6 mmol/l, n (\%) & 79 (28.6) & $63(28)$ & $16(33)$ & 0.43 \\
\hline Peak blood ketones, mmol/l & $0.8(0.3,1.9)$ & $0.85(0.2,1.9)$ & $0.65(0.3,1.0)$ & 0.32 \\
\hline Worst ketosis, n (\%) & & & & 0.52 \\
\hline No ketosis $(<0.6 \mathrm{mmol} / \mathrm{l})$ & $22(36.7)$ & $16(35)$ & $6(42)$ & \\
\hline Mild (0.6-1.5 mmol/l) & $20(33.3)$ & $15(33)$ & $5(36)$ & \\
\hline Moderate (1.6-3.0 mmol/l) & $11(18.3)$ & $8(17)$ & $3(21)$ & \\
\hline Marked (>3.0 mmol/l) & $7(11.7)$ & $7(15)$ & 0 & \\
\hline Number of urine ketone measurements & 261 & 215 & 46 & \\
\hline Worst ketonuria, n (\%) & & & & 0.51 \\
\hline No ketonuria & $40(66.7)$ & $31(67)$ & $9(64)$ & \\
\hline Mild (1+) & $13(21.7)$ & $10(22)$ & $3(21)$ & \\
\hline Moderate $(2+)$ & $6(10)$ & $5(11)$ & $1(7)$ & \\
\hline Marked (3+) & $1(1.7)$ & 0 & $1(7)$ & \\
\hline Patients with alkalosis ${ }^{\mathrm{a}}, \mathrm{n}(\%)$ & $25(42)$ & $20(43)$ & $5(36)$ & 0.76 \\
\hline \multicolumn{5}{|l|}{ Blood gas analysis, worst value during study period } \\
\hline $\mathrm{pH}$ & $7.4(7.3,7.4)$ & $7.3(7.3,7.4)$ & $7.4(7.3,7.4)$ & 0.32 \\
\hline Lactate, $\mathrm{mmol} / \mathrm{l}$ & $2.2(1.6,3.1)$ & $2.3(1.7,3.4)$ & $2(1.4,2.3)$ & 0.15 \\
\hline Bicarbonate, mmol/l & $22(20,24)$ & $22(19,24)$ & $25(23,26)$ & 0.002 \\
\hline Chloride, $\mathrm{mmol} / \mathrm{l}$ & $108(104,111)$ & $108(104,111)$ & $105(102,108)$ & 0.10 \\
\hline Sodium, mmol/l & $136(132,138)$ & $136(132,138)$ & $136(132,136)$ & 0.73 \\
\hline Albumin, $g / l$ & $26(22,30)$ & $26(21,29)$ & $29(23,30)$ & 0.75 \\
\hline
\end{tabular}


Table 2 Glycemic control, ketonemia, ketonuria and acid-base status of diabetic patients with and without permissive hyperglycemia (Continued)

\begin{tabular}{|c|c|c|c|c|}
\hline Base excess (BE), mmol// & $-2.8(-5,0)$ & $-3(-5,-1)$ & $0.35(-4,1)$ & 0.006 \\
\hline ADA ketoacidosis, n (\%) & $2 / 60(3.3)$ & $1 / 46(2.2)$ & $1 / 14(7.1)$ & 1.00 \\
\hline JBDS ketoacidosis, n (\%) & $0 / 60(0)$ & & & \\
\hline
\end{tabular}

Values are median (IQR) or $\mathrm{n}(\%)$

$B G L$ blood glucose level, ICU intensive care unit, ADA American Diabetes Association, JBDS Joint British Diabetes Society ${ }^{\mathrm{a}} \mathrm{pH}>7.45$

[33 \%] mild, 11 [18 \%] moderate and seven [12\%] severe ketosis). A total of 20 (33\%) patients had some degree of ketonuria (13 [22\%] mild, six [10\%] moderate and one [2\%] severe ketonuria). Additionally, 25 (42\%) patients had at least one episode of alkalosis ( $\mathrm{pH}>7.45)$. On crude comparison, we observed a similar prevalence and severity of ketosis and ketonuria in patients with peak BGL above and below $10 \mathrm{mmol} / \mathrm{l}$. In contrast, patients with a peak BGL $\geq 10 \mathrm{mmol} / \mathrm{l}$ had lower bicarbonate levels $(P=0.001)$ and base excess $(P=0.006)$.

However, only two out of 60 (3.3\%) patients (one patient in each group) fulfilled the criteria for ketoacidosis according to the JBDS consensus criteria (no patients developed ADA-defined ketoacidosis, Table 2). A description of these two individuals is provided in the Additional file 1.

Patients with $(\mathrm{n}=38)$ and without $(\mathrm{n}=22)$ ketosis had similar HbA1c levels (median HbA1c $6.7 \%$ in both groups, $P=0.72$ ) whereas admission hematocrit levels were higher in patients with ketosis $(P=0.046)$. A similar proportion of patients had insulin-dependent diabetes in the two groups (42 vs. $50 \%$ ). Moreover, admission and peak BGLs did not differ significantly (Additional file 1: Table S1 and S2).

In contrast, compared to patients with mild to moderate ketosis $(\mathrm{n}=31)$, insulin-dependent diabetes tended to be more common (71 \% vs. $35 \%, P=0.12)$, HbAlc was significantly greater $(8.2 \%$ vs. $6.5 \%, P<0.001)$ and admission hematocrit was lower $(25 \%$ vs. $30 \%, P=0.03$ ) among patients with severe ketosis $(\mathrm{n}=7)$ (Additional file 1: Table S3).

In patients with $\mathrm{BGL} \geq 10 \mathrm{mmol} / \mathrm{l}$, ketosis resolved within 3-4 days irrespective of whether insulin was administered or not (Fig. 1a) and despite persistent hyperglycemia according to protocol (Fig. 1b).

We found no significant correlation between admission blood ketone levels and admission BGLs (Fig. 2a), HbA1c levels (Fig. 2b), blood lactate levels (Fig. 2c), hematocrit levels (Fig. 2d), or catecholamine infusion (Fig. 2e) in patients not receiving insulin $(n=43)$. In contrast, patients in the mid-APACHE III tertile had lower admission blood ketone levels than patients in the highest and lowest tertile (Fig. 2f). Patients with alkalosis on admission had significantly lower blood ketone levels than patients without alkalosis $(P=0.04)$ whereas patients admitted following cardiopulmonary bypass surgery had significantly greater blood ketone levels (median [IQR] $0.6[0.2,1.3] \mathrm{mmol} / \mathrm{l}$ vs. $0.2[0.1,0.7] \mathrm{mmol} / \mathrm{l}$ than patients without cardiopulmonary bypass surgery, $P=0.049$ ). On multivariable linear regression analysis, restricted to patients not receiving insulin $(n=43)$, we observed no independent association between blood ketone levels and blood glucose levels, HbA1c levels, blood lactate levels, hematocrit levels,

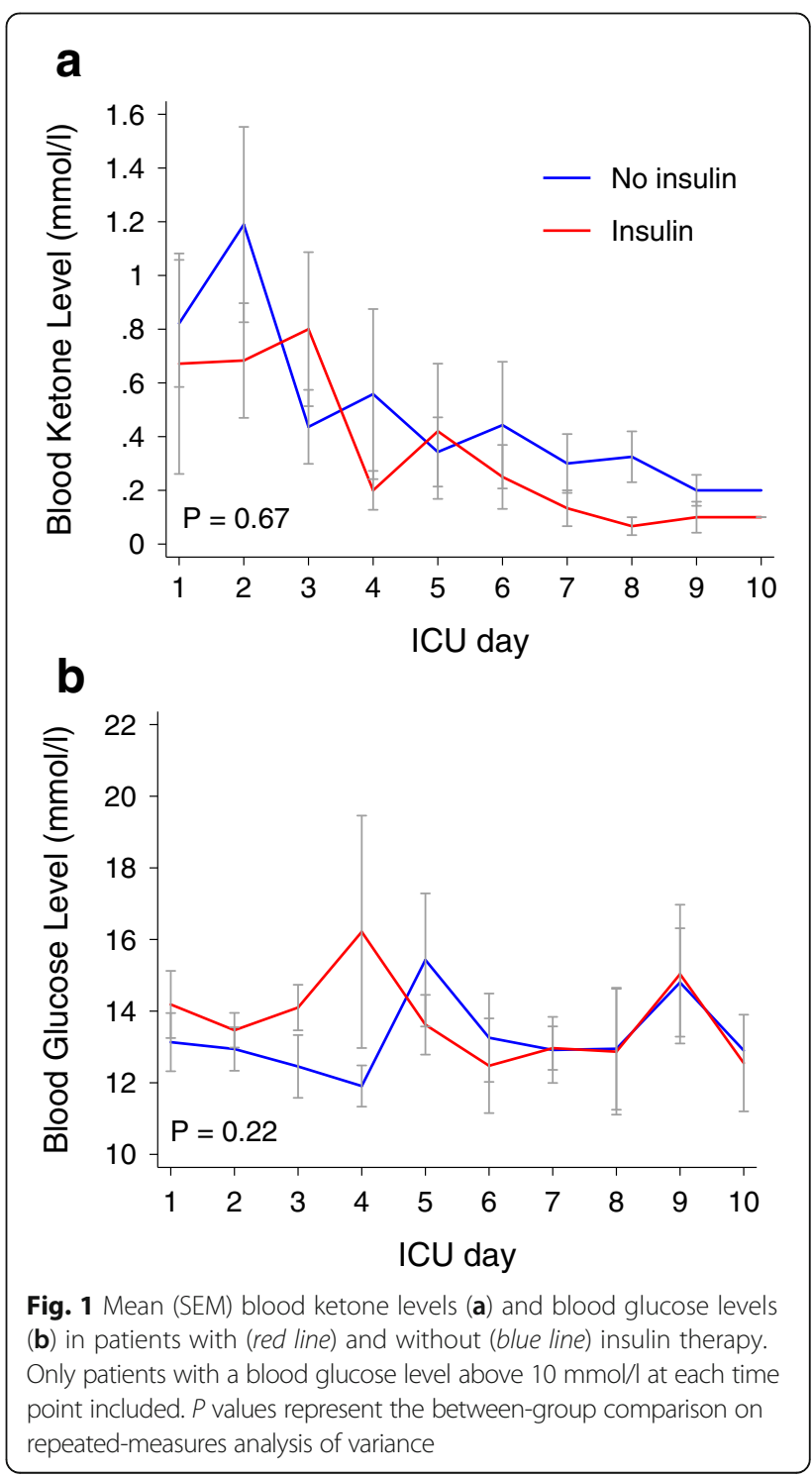



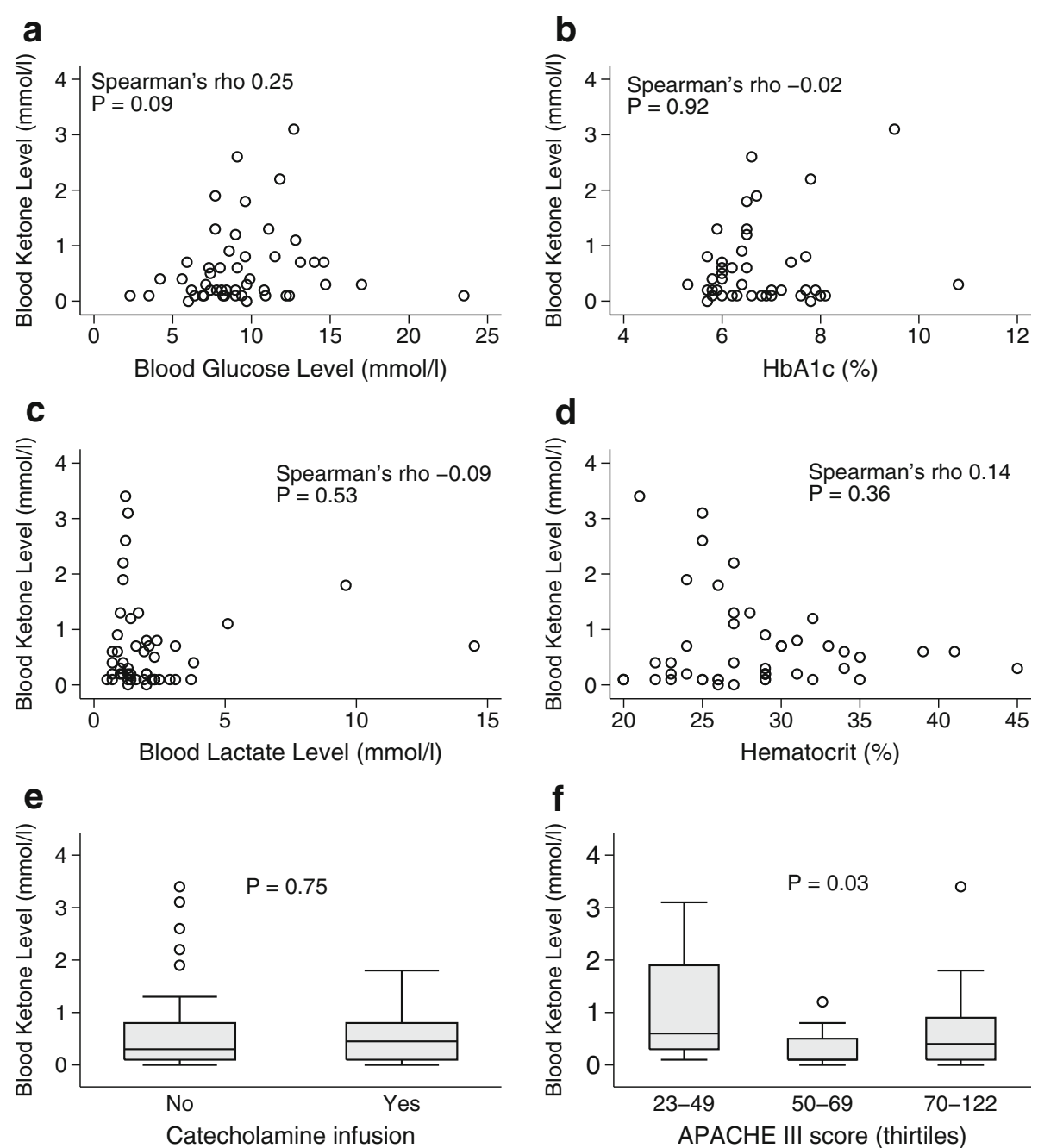

Fig. 2 Correlation between blood ketone levels and blood glucose levels (a), HbA1c (b), lactate (c), hematocrit (d), catecholamine infusion (e) and APACHE III quartiles (f) on ICU admission in patients not receiving insulin

catecholamine infusion or APACHE III score. However, a trend towards greater blood ketone levels in patients admitted following cardiopulmonary bypass was observed in the adjusted analysis $(P=0.06)$ (Table 3$)$.

\section{Discussion}

\section{Key findings}

We performed a prospective observational study to explore the prevalence of ketonemia and ketonuria in critically ill diabetic patients treated within a liberal glycemic control protocol. Overall, about two thirds of our patients had some degree of ketosis $(\beta-\mathrm{OHB} \geq 0.6 \mathrm{mmol} / \mathrm{l}$ ) during their ICU stay and $33 \%$ had some degree of ketonuria. However, ketosis in these critically ill diabetic patients managed with liberal glycemic control was typically seen in the first 24-48 hours of admission and appeared independent of glucose control, HbA1c level and severity of illness. Moreover, ketosis resolved in all patients irrespective of whether insulin was given or not. Finally, ketosis appeared more pronounced following cardiopulmonary bypass surgery.

\section{Relationship to previous studies}

To our knowledge, this is the first study investigating the prevalence of ketosis in critically ill diabetic patients without acute diabetic ketoacidosis or hyperosmolar hyperglycemic state. We are also the first to describe the prevalence of ketosis in patients treated with a liberal glucose protocol (BGL target $10-14 \mathrm{mmol} / \mathrm{l}$ ).

In accordance with our results, a study of 32 ventilated non-diabetic ICU patients with $(\mathrm{n}=19)$ and without $(\mathrm{n}=$ 13) stress hyperglycemia (BGL up to $16 \mathrm{mmol} / \mathrm{l}$ ) found no relationship between breath acetone concentration and BGL [17]. While in most patients $(n=11)$ breath acetone concentration decreased after insulin administration, some had persistent ketosis despite normal BGL. 
Table 3 Multivariable linear regression analysis showing the association with admission blood ketone level (mmol/l) in patients not receiving insulin

\begin{tabular}{|c|c|c|c|c|}
\hline \multirow[b]{2}{*}{$\underline{\text { Variable }}$} & \multicolumn{2}{|l|}{ Univariable analysis } & \multicolumn{2}{|l|}{ Multivariable analysis } \\
\hline & estimate $(95 \%$ Cl) & $P$ value & estimate $(95 \% \mathrm{Cl})$ & $P$ value \\
\hline Blood glucose level, per mmol/l & $0.03(-0.04$ to 0.10$)$ & 0.35 & $0.03(-0.05$ to 0.12$)$ & 0.44 \\
\hline HbA1c, per \% & $0.16(-0.08$ to 0.40$)$ & 0.18 & $0.16(-0.10$ to 0.42$)$ & 0.21 \\
\hline Blood lactate, per mmol/l & $0.02(-0.08$ to 0.12$)$ & 0.72 & $0.05(-0.08$ to 0.19$)$ & 0.41 \\
\hline Hematocrit, per \% & $-0.02(-0.06$ to 0.03$)$ & 0.38 & $-0.03(-0.08$ to 0.01$)$ & 0.15 \\
\hline \multicolumn{5}{|l|}{ Catecholamine infusion } \\
\hline No & Referent & & Referent & \\
\hline Yes & $-0.19(-0.67$ to 0.29$)$ & 0.43 & $-0.57(-1.30$ to 0.16$)$ & 0.12 \\
\hline \multicolumn{5}{|l|}{ Cardiopulmonary bypass surgery } \\
\hline No & Referent & & Referent & \\
\hline Yes & $0.44(-0.02$ to 0.91$)$ & 0.06 & $0.67(-0.03$ to 1.37$)$ & 0.06 \\
\hline \multicolumn{5}{|l|}{ APACHE III score (tertile) } \\
\hline $23-49$ & Referent & & Referent & \\
\hline $50-69$ & $-0.68(-1.23$ to -0.13$)$ & 0.02 & $-0.41(-1.14$ to 0.33$)$ & 0.27 \\
\hline $70-122$ & $-0.30(-0.85$ to 0.25$)$ & 0.28 & $-0.29(-0.54$ to 1.12$)$ & 0.49 \\
\hline
\end{tabular}

HbA1c glycated hemoglobin A1c, APACHE Acute Physiology and Chronic Health Evaluation

The two main ketone bodies, acetoacetate (AcAc) and 3-beta-hydroxybutyrate $(\beta-\mathrm{OHB})$, usually circulate in low concentrations $(<0.5 \mathrm{mmol} / \mathrm{l})$ in healthy adults [18]. Serum levels of $\beta$-OHB start to rise after 12-16 hours of fasting (an essentially ubiquitous state in critically ill patients in the period surrounding ICU admission), reaching 1-2 $\mathrm{mmol} / \mathrm{l}$ after 2 days [19-21], and $6-8 \mathrm{mmol} / \mathrm{l}$ after 2-3 weeks of starvation [22, 23].

Harano et al. [13] compared serum ketone levels between non-acutely ill type $1(\mathrm{n}=117)$ and type $2(\mathrm{n}=$ 260) diabetic patients and 91 healthy subjects after an overnight fast. In patients with type 1 diabetes, ketone bodies were higher despite normal fasting BGL and increased proportionally to the BGL in hyperglycemic individuals. In type 2 diabetics, however, the authors found only a weak correlation between fasting plasma glucose and ketonemia with serum ketone levels rarely exceeding $2 \mathrm{mmol} / \mathrm{l}$. Interestingly, a post-prandial reduction in 3-hydroxybutyrate was noted in subjects with a sufficient endogenous insulin response (on dietary control or sulphonylureas), but not in subjects with insulindependent type 2 diabetes, who are also known to have greater ketogenesis after nighttime fasting (12). Duska et al. [24] explored the relative impact of exogenous insulin administration and endogenous insulin secretion on plasma insulin levels in critically ill trauma patients without diabetes. They found that, in such patients without beta-cell dysfunction, the plasma insulin level is mainly determined by the endogenous secretion rather than by the insulin infusion, even during the acute phase when exogenous insulin requirements were high.
Preserved beta-cell function with sufficient endogenous insulin secretion could also explain the relatively low blood ketone levels in our cohort and the similar ketone kinetics in hyperglycemic patients with and without exogenous insulin administration. Moreover, among the few patients with marked ketonemia $(n=7)$ the majority was insulin-dependent suggesting significant beta-cell failure. However, even in this subgroup ketonemia resolved despite permissive hyperglycemia.

The effect of fasting on urine ketone concentration has been studied in a large Korean trial of 16,523 volunteers [25]. Only $8.8 \%$ of all subjects developed ketonuria after fasting overnight. Among those without ketonuria, the prevalence of obesity and metabolic syndrome was significantly higher and cholesterol levels, insulin levels and body mass index (BMI) were more likely to be abnormal. A ketotic response to fasting could, therefore, indicate a favorable metabolic state.

In addition to fasting, ketosis (and ketoacidosis) may be caused by excessive alcohol consumption, severe hypoxia, counter-regulatory hormone excess, catecholamine infusion, parenteral nutrition, alkalosis, severe liver disease, and various inborn errors of metabolism $[8,9,11$, $18,26,27]$. However, among our patients with acute liver failure $(\mathrm{n}=3)$, chronic alcohol intake $(\mathrm{n}=4)$ and acute alcohol intoxication $(\mathrm{n}=1)$ only one patient (acute-on-chronic liver failure) developed ketosis (peak blood $\beta$-OHB level $1.4 \mathrm{mmol} / \mathrm{l})$. Furthermore, catecholamine infusion or alkalosis was not associated with elevated $\beta$-OHB levels in our study. 
In contrast, in our patients, cardiopulmonary bypass surgery (performed during normothermia) appeared to be an important trigger of ketogenesis. This finding is supported by Hashimoto et al. [12]. In 24 patients, they compared the arterial acetoacetate to $\beta$-OHB ratio (the so-called ketone body ratio) between patients undergoing normothermic vs. hypothermic cardiopulmonary bypass surgery. The ratio decreased significantly in both groups suggesting reduced mitochondrial redox potential favoring conversion to $\beta$ $\mathrm{OHB}$ in such patients. Importantly, the decrease in ketone body ratio was more pronounced after hypothermic bypass indicating even greater $\beta$-OHB generation in that group.

\section{Implications of study findings}

Our finding that a BGL $\geq 10 \mathrm{mmol} / \mathrm{l}$ is not associated with increased ketogenesis and that critical illness is associated with some degree of peri-admission ketosis irrespective of blood glucose level and insulin therapy implies that a BGL range of $10-14 \mathrm{mmol} / \mathrm{l}$ in critically ill diabetic patients may be associated with minimal additional ketogenic metabolic stress.

Low nutritional intake in the hours and days before ICU admission is a plausible explanation for the observed early ketosis of critical illness in our population. Indeed, although only about half of our patients received nutrition, their caloric intake progressively increased, which may have contributed to the observed decline in ketone levels over time. In addition, it seems that endogenous insulin secretion due to residual betacell function may be sufficient to suppress ketogenesis in patients not requiring insulin. This hypothesis is also supported by a lack of correlation between the level of ketonemia and blood glucose in our patients. In addition, even in our patients with premorbid insulindependent diabetes, the majority of which also required insulin in ICU, early ketosis resolved despite sustained hyperglycemia.

The clinical relevance of ketosis in ICU patients remains unclear as recent studies suggest that $\beta$ OHB could have anti-inflammatory effects by inhibiting interleukin (IL)-1 $\beta$ and IL-18 production [28]. In addition, ketone bodies provide important energy source for vital organs not only during starvation but also during stress-induced insulin resistance. Ketogenesis could, therefore, be an adaptive and protective response to critical illness.

\section{Strengths and limitations}

Our study has several strengths. We prospectively analyzed a representative sample of patients with diabetes using the same point-of-care analysis platform in every patient. The setting of a tertiary hospital with a heterogeneous case mix is likely to reflect the average ICU population. We performed daily ketone measurements and simultaneously tested urine samples for ketonuria during the entire ICU stay or up to 10 consecutive days. We used a multivariable model to assess the relationship between blood ketone levels and glycemic control, markers of disease severity and insulin administration. Our findings are robust and demonstrate that blood ketone levels are unrelated to blood glucose levels or insulin treatment in the critically ill diabetic patient without diabetic ketoacidosis within the boundaries of a liberal glucose protocol.

Our study has some limitations. First, it is observational in nature and not a randomized controlled study. However, our aim was to explore the prevalence of ketosis during liberal glucose management of critically ill diabetic patients. Second, this is a single-center study with only a limited number of patients. However, we used solid methodology and obtained more than 270 blood ketone measurements and analyzed more than 100 hyperglycemic episodes. Third, we used a semi-quantitative method to measure ketonuria. However, given the inherent limitations $[18,27]$ of pointof-care urine ketone tests, and in view of our results, it is unlikely that quantitative methods would have influenced our results. Yet, previous validation studies suggest that the use of point-of-care instruments may overestimate true blood $\beta$-OHB levels when hematocrit is below normal [14], which was the case in most of our patients. Hence, we may have overestimated the true ketosis prevalence. Fourth, we did not measure plasma insulin concentrations or Cpeptide levels and were therefore not able to quantify betacell function. Furthermore, although we lack data on counter-regulatory hormone, FFA, amino acid and oxaloacetate levels, we assessed clinical variables relevant to ketogenesis. Fifth, we did not measure glycosuria, which may be particularly pronounced in critically ill patients during permissive hyperglycemia. The degree of glycosuria in such patients and its impact on patient-centered outcomes should therefore be explored in future studies. Sixth, we did not measure acetoacetate and acetone and could therefore not quantify the total ketotic activity. Perturbations in the mitochondrial redox state during critical illness may alter the ratio between acetoacetate and $\beta-\mathrm{OHB}$ [29]. However, we performed repeated $\beta-\mathrm{OHB}$ measurements over several days and observed a consistent and marked decline over time. It is unlikely that unmeasured ketones would have a different directional change over the corresponding time frame. Finally, because the results of the daily ketone measurements were documented on the observational chart, we cannot exclude that these data influenced glucose management by clinicians. However, nursing and research staff performed ketone measurements and documentation without direct involvement of medical staff.

\section{Conclusions}

Ketosis in critically ill diabetic patients is common and typically occurs in the period early after admission. Ketosis 
may be particularly pronounced following cardiopulmonary bypass surgery. However, in critically ill diabetic patients, liberal glycemic control or insulin therapy does not appear to be associated with ketosis. These findings suggest that liberal glycemic control in these patients does not cause additional ketotic stress and imply that factors beyond glycemic control and insulin therapy may be more important drivers of ketosis in such patients.

\section{Additional file}

Additional file 1: Table S1. Baseline characteristics of diabetic patients with and without ketosis. Table S2. Glycemic control in diabetic patients with and without ketosis. Table S3. Baseline characteristics of patients with mild or moderate and marked ketosis. Description of the two patients with American Diabetes Association (ADA) ketoacidosis. Figure S1. Change in hematocrit over time for all study patients. Figure S2. Daily mean caloric intake in patients receiving nutrition. (DOCX $90 \mathrm{~kb}$ )

\section{Abbreviations \\ ABG: arterial blood gas; AcAc: acetoacetate; ADA: American Diabetes Association; APACHE: Acute Physiology and Chronic Health Evaluation; BGL: blood glucose level; $\beta$-OHB: 3-beta-hydroxybutyrate; DKA: diabetic ketoacidosis; FFA: free fatty acids; HbA1c: glycated hemoglobin A1c; HHS: hyperglycemic hyperosmolar state; ICU: intensive care unit; IL: interleukin; JBDS: Joint British Diabetes Society; RM-ANOVA: repeated- measures analysis of variance; RRT: renal replacement therapy}

\section{Acknowledgements}

The authors would like to thank our research coordinators Mrs. Leah Peck and Ms. Helen Young for their great help and support with realizing this study.

\section{Funding}

Austin Hospital Intensive Care Trust Fund

Role of sponsor/funder/institution in developing protocol: none

\section{Availability of supporting data}

The dataset analysed during the current study is available from the corresponding author on reasonable request.

\section{Authors' contributions}

NL made substantial contributions to study design, acquisition, analysis and interpretation of data and has been involved in drafting and revising the manuscript. LC made substantial contributions to acquisition of data, analysis and interpretation of data and has been involved in drafting and revising the manuscript. MC made substantial contributions to acquisition of data and was involved in drafting the manuscript. RB made substantial contributions to conception and design of the study and has been involved in revising the manuscript critically for important intellectual content. GE made substantial contributions to the study design and acquisition of data and has been involved in drafting and revising the manuscript. JM made substantial contributions to study design, analysis and interpretation of data and has been involved in revising the manuscript critically for important intellectual content. All authors read and approved the final manuscript and take public responsibility for the content.

\section{Competing interests}

The authors declare that they have no competing interests.

\section{Consent for publication}

Not applicable

\section{Ethics approval and consent to participate}

The Austin Health Human Research Ethics Committee approved the study (approval number LNR/14/Austin/487, HREC 131217 and 120712) with a waiver for informed consent.

\section{Author details}

'Department of Intensive Care, Austin Hospital, 145 Studley Rd, Heidelberg 3084, VIC, Australia. ${ }^{2}$ Department of Intensive Care Medicine, Lucerne Cantonal Hospital, Lucerne, Switzerland. ${ }^{3}$ Department of Perioperative Medicine, Intensive Care and Emergency, Cattinara Hospital, Trieste University School of Medicine, Trieste, Italy. ${ }^{4}$ Australian and New Zealand Intensive Care Research Centre (ANZIC-RC), Department of Epidemiology and Preventive Medicine, Monash University, Melbourne, VIC, Australia. ${ }^{5}$ Department of Anaesthesia and Intensive Care Medicine, Karolinska University Hospital, Department of Physiology and Pharmacology, Karolinska Institutet, 17177 Stockholm, Sweden.

Received: 29 May 2016 Accepted: 22 August 2016

Published online: 15 September 2016

\section{References}

1. Griesdale DE, de Souza RJ, van Dam RM, Heyland DK, Cook DJ, Malhotra A et al. Intensive insulin therapy and mortality among critically ill patients: a meta-analysis including NICE-SUGAR study data. CMAJ. 2009;180(8):821-7. doi:10.1503/cmaj.090206.

2. Finfer S, Chittock DR, Su SY, Blair D, Foster D, Dhingra V, et al. Intensive versus conventional glucose control in critically ill patients. N Engl J Med. 2009;360(13):1283-97. doi:10.1056/NEJMoa0810625.

3. Egi M, Finfer S, Bellomo R. Glycemic control in the ICU. Chest. 2011;140(1):212-20. doi:10.1378/chest.10-1478.

4. Dellinger RP, Levy MM, Rhodes A, Annane D, Gerlach H, Opal SM, et al. Surviving Sepsis Campaign: international guidelines for management of severe sepsis and septic shock, 2012. Intensive Care Med. 2013;39(2):165-228.

5. Egi M, Krinsley JS, Maurer P, Amin DN, Kanazawa T, Ghandi S, et al. Premorbid glycemic control modifies the interaction between acute hypoglycemia and mortality. Intensive Care Med. 2016;42(4):562-71. doi:10.1007/s00134-016-4216-8.

6. Egi M, Bellomo R, Stachowski E, French CJ, Hart GK, Taori G, et al. The interaction of chronic and acute glycemia with mortality in critically ill patients with diabetes. Crit Care Med. 2011;39(1):105-11. doi:10.1097/CCM. 0b013e3181feb5ea.

7. Di Muzio F, Presello B, Glassford NJ, Tsuji IY, Eastwood GM, Deane AM, et al. Liberal versus conventional glucose targets in critically ill diabetic patients: an exploratory safety cohort assessment. Crit Care Med. 2016;44(9):1683-91. doi:10.1097/ccm.0000000000001742.

8. Keller U, Gerber PP, Stauffacher W. Stimulatory effect of norepinephrine on ketogenesis in normal and insulin-deficient humans. Am J Physiol. 1984;247(6 Pt 1):E732-9.

9. Ball MJ. Parenteral nutrition in the critically ill: use of a medium chain triglyceride emulsion. Intensive Care Med. 1993;19(2):89-95.

10. White $\mathrm{H}$, Venkatesh B. Clinical review: ketones and brain injury. Crit Care. 2011;15(2):219. doi:10.1186/cc10020.

11. Lipsky SR, Apler BJ, Rubini ME, Van Eck WF, Gordon ME. The effects of alkalosis upon ketone body production and carbohydrate metabolism in man. J Clin Invest. 1954;33(9):1269-76. doi:10.1172/jci103002.

12. Hashimoto K, Sasaki T, Hachiya T, Onoguchi K, Takakura H, Oshiumi M, et al. Superior hepatic mitochondrial oxidation-reduction state in normothermic cardiopulmonary bypass. J Thorac Cardiovasc Surg. 2001;121(6):1179-86. doi:10.1067/mtc.2001.113599.

13. Harano Y, Kosugi K, Hyosu T, Suzuki M, Hidaka H, Kashiwagi A, et al. Ketone bodies as markers for type 1 (insulin-dependent) diabetes and their value in the monitoring of diabetic control. Diabetologia. 1984;26(5):343-8.

14. Ceriotti F, Kaczmarek E, Guerra E, Mastrantonio F, Lucarelli F, Valgimigli F, et al. Comparative performance assessment of point-of-care testing devices for measuring glucose and ketones at the patient bedside. J Diabetes Sci Technol. 2015;9(2):268-77. doi:10.1177/1932296814563351.

15. Kitabchi AE, Umpierrez GE, Miles JM, Fisher JN. Hyperglycemic crises in adult patients with diabetes. Diabetes Care. 2009;32(7):1335-43. doi:10.2337/dc09-9032.

16. Savage MW, Dhatariya KK, Kilvert A, Rayman G, Rees JA, Courtney CH, et al. Joint British Diabetes Societies guideline for the management of diabetic ketoacidosis. Diabet Med. 2011;28(5):508-15. doi:10.1111/j.1464-5491.2011.03246.x.

17. Sturney SC, Storer MK, Shaw GM, Shaw DE, Epton MJ. Off-line breath acetone analysis in critical illness. J Breath Res. 2013;7(3):037102. doi:10.1088/1752-7155/7/3/037102.

18. Laffel L. Ketone bodies: a review of physiology, pathophysiology and application of monitoring to diabetes. Diabetes Metab Res Rev. 1999;15(6):412-26. 
19. Robinson AM, Williamson DH. Physiological roles of ketone bodies as substrates and signals in mammalian tissues. Physiol Rev. 1980;60(1):143-87.

20. Cahill Jr GF, Herrera MG, Morgan AP, Soeldner JS, Steinke J, Levy PL, et al. Hormone-fuel interrelationships during fasting. J Clin Invest. 1966;45(11):1751-69. doi:10.1172/jci105481.

21. Hall SE, Wastney ME, Bolton TM, Braaten JT, Berman M. Ketone body kinetics in humans: the effects of insulin-dependent diabetes, obesity, and starvation. J Lipid Res. 1984;25(11):1184-94.

22. Newman JC, Verdin E. Ketone bodies as signaling metabolites. Trends Endocrinol Metab. 2014;25(1):42-52. doi:10.1016/j.tem.2013.09.002.

23. Cahill Jr GF. Fuel metabolism in starvation. Annu Rev Nutr. 2006;26:1-22. doi:10.1146/annurev.nutr.26.061505.111258.

24. Duska F, Andel M. Intensive blood glucose control in acute and prolonged critical illness: endogenous secretion contributes more to plasma insulin than exogenous insulin infusion. Metab Clin Exp. 2008;57(5):669-71. doi:10.1016/j.metabol.2008.01.001.

25. Joo NS, Lee DJ, Kim KM, Kim BT, Kim CW, Kim KN, et al. Ketonuria after fasting may be related to the metabolic superiority. J Korean Med Sci. 2010;25(12):1771-6. doi:10.3346/jkms.2010.25.12.1771.

26. Schade DS, Eaton RP. Differential diagnosis and therapy of hyperketonemic state. JAMA. 1979;241(19):2064-5.

27. MacGillivray MH, Li PK, Lee JT, Mills BJ, Voorhess ML, Putnam Tl, et al. Elevated plasma beta-hydroxybutyrate concentrations without ketonuria in healthy insulin-dependent diabetic patients. J Clin Endocrinol Metab. 1982;54(3):665-8. doi:10.1210/jcem-54-3-665.

28. Youm YH, Nguyen KY, Grant RW, Goldberg EL, Bodogai M, Kim D, et al. The ketone metabolite beta-hydroxybutyrate blocks NLRP3 inflammasome-mediated inflammatory disease. Nat Med. 2015;21(3):263-9. doi:10.1038/nm.3804.

29. Yassen KA, Galley HF, Lee A, Webster NR. Mitochondrial redox state in the critically ill. Br J Anaesth. 1999;83(2):325-7.

\section{Submit your next manuscript to BioMed Central and we will help you at every step:}

- We accept pre-submission inquiries

- Our selector tool helps you to find the most relevant journal

- We provide round the clock customer support

- Convenient online submission

- Thorough peer review

- Inclusion in PubMed and all major indexing services

- Maximum visibility for your research

Submit your manuscript at www.biomedcentral.com/submit

) Biomed Central 